NASA Technical Memorandum 100165

\title{
Aeroacoustics of Subsonic Turbulent Shear Flows
}

Marvin E. Goldstein

Lewis Research Center

Cleveland, Ohio

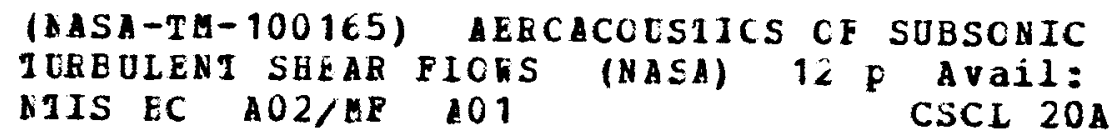

N $87-2 \in \in 15$

IUREULENI SHEAR FICRS (NASA) 12 P AVail:
NIIS EC AO2/MP 01

$63 / 71 \quad \begin{array}{ll}\text { Unclas } \\ 00906 \vdots 7\end{array}$

Prepared for the

11th Aeroacoustics Conference

sponsored by the American Institute of Aeronautics and Astronautics

Sunnyvale, California, October 19-21, 1987

\section{nNSA}




\title{
AEROACOUSTICS OF SUBSONIC TURBULENT SHEAR FLOWS
}

\author{
Marvin E. Goldstein* \\ National Aeronautics and Space Administration \\ Lewis Research Center \\ Cleveland, ohio
}

\section{Abstract}

The paper is a review of sound generation in turbulent shear flows. It emphasizes simultaneous calculation of the turbulent flow along with the resulting sound generation rather than the alternative acoustic analogy approach. The first part of the paper is concerned with solid surface interaction. The second part concentrates on the sound generated by turbulence interacting with itself.

\section{Introduction}

The subject of this paper became a serious scientific discipline about 35 years ago when Lighthili26,27 published $h$ is acoustic analogy theory of jet notse. That work has more or less dominated the subsequent development of this subject, which is still somewhat incomplete--even though it has undergone little change in the past five years or so. This paper does not place much emphasis on the acoustic analogy but rather concentrates on an alternative approach, which may be more readily adapted for use on large-scale computers to obtain more detalled information about the sound field than would be possible from the acoustic analogy.

This approach amounts to little more than calculating the unsteady flow that produces the sound simultaneousiy with the resulting sound field, starting from some prescribed upstream state that is idealiy specified just ahead of this region where the sound generation takes place. To make progress without resorting to full-scale numerical computation requires that the governing equations be linearized about some appropriate mean flow. But that ultimately has to be done, elther implicitiy or explicitiy, even with the acoustic analogy approach. I have no doubt that the day will come when turbulence-generated sound is calculated directly from the Navier-Stokes equation, but, to my knowledge, that has yet to be done, and there is much to be learned from the existing work, which has often led to relatively simple formulas that show encouraging agreement with experiment and produce a great deal of insight into and physical understanding of the sound generation process. This paper is a very selective review of that work, and it adopts a very specific viewpoint.

The use of linearized theory to calculate turbulent flows or, better yet, changes in turbulent flows is a branch of turbulence theory now known as "rapid-distortion theory" (see Moffatt ${ }^{31}$ ). It assumes that the following assumptions are satisfied (Hunt ${ }^{24}$ ): (a) that $u^{\prime} / U \ll 1$, where $u^{\prime}$ is the rms turbulence velocity and $U$ is the local meanflow velocity; and (b) that the interaction or change being calculated be completed in a time, t I say, that is short compared with rdecay, where rdecay is the decay time or lifetime of a typical turbulent eddy $0\left(\ell / u^{\prime}\right)--\ell$ being the characteristic size of turbulent eddies. Rapid-distortion calculations are

*Fellow AIAA. usually based on the inviscid equations-- an approximation that is justified when both the mean flow and turbulence Reynolds numbers are large. The important point here is that the radiated sound field can be determined as a by-product of any such rapid-distortion-theory calculation, as long as compressibility effects are retained.

While it might seem most logical to begin by omitting solid surface effects and to include them only after the turbulence self-notse problem has been appropriately dealt with, it turns out that the solid boundaries actually simplify the problem and allow a more rigorous treatment in, at least, some cases. Consider then a high-Reynolds-number turbulent airjet such as shown schematically in Fig. 1. The maximum turbulence level occurs along the centerline of the inftial mixing layer, indicated by the dashed line in the figure. Here the ratio of the rms turbulence velocity to the local mean-flow velocity is roughly equal to 0.24 (Bradshaw et al. ${ }^{5}$ ) which, although not all that small, would probably still be considered to be an acceptable "small parameter" to many classical applied mathematicians. Condition (a) is therefore reasonably well satisfled.

\section{Solid Surface Effects}

Now suppose that a semi-infinite but infinitesimally thin flat plate is inserted into the flow as shown in the figure. Then the interaction between the turbulence and the leading edge will be completed in a time $\tau_{I}=O(2 / U)$, which, in view of the smallness of the turbulence intensity, is fairly small compared with $\tau_{\text {decay }}=0\left(\ell / u^{\prime}\right)$. Thus, inviscid rapid-distortion theory applies, and the interaction between the turbulence and the edge can be calculated by linearizing the inviscid equations (the Euler equations) about the mean flow.

Since the ratio of the cross-stream to streamwise components of the mean-flow velocity is of the order of $\left(u^{\prime} / U\right)^{2}$ (Tennekes and Lumley ${ }^{40}$ ), the order of approximation will certainly not be diminished if this flow is taken to be a unidirectional transverseiy sheared flow. The important advantage of using this flow is that it is itself a solution of the inviscid equations (for any velocity profile). The resulting expansion is then a rational perturbation that can, in principie, be carried to arbitrary order without internal inconsistency. The lowest order equations are the same as those used in inviscid stability theory, 1.e. the Rayleigh equations (see Betchov and Criminale ${ }^{4}$ ), and as already indicated, the radiated sound field can be determined as part of the solution to these equations--provided, of course, that compressibility effects are retained.

To accomplish this, one must first decide on an approprlate representation of the incident turbulence. This would be rather easy to do if the mean flow were completely uniform, since any solution for the unsteady velocity/pressure fluctuations could then be decomposed into the sum of an 
"acoustic solution" that carries no vorticity and $\dot{a}$ vortical solution which produces no pressure fluctuations and which is often referred to as the "gust" or "hydrodynamic" solution. The latter is used to represent the incident turbulence in most problems that involve the interaction of turbulence with solid surfaces embedded in uniform mean flow. Its suttability for this purpose is largely due to the following reasons:

(1) It does not become infinite anywhere in space, even in the absence of solid surfaces, so that it can describe the turbulence field that would exist if the surfaces were not present.

(2) It involves two arbitrary "convected" quantities that can be specified as upstream boundary conditions to describe the turbulence entering the interaction zone in any given problem. This seems to be the appropriate degree of generality, because the the vorticity is a convected quantity that has only two independent components (since its divergence must vanish).

(3) It has no acoustic radiation field at subsonic speeds and will in fact vanish exponentialiy fast at transverse infinity if the mean and unsteady vorticity fields are suffictently compact.

Decomposition of the solution into acoustic and vortical parts is no longer possible when the mean flow is nonuniform, but the compressible Rayleigh equations still possess a solution that has the three properties listed above and, in fact, approaches the "vortical" solution on a uniform mean flow in the limit as the mean flow approaches a uniform flow (Goldstein 18,19 and Mohring 32 ). This would then seem to be the natural generalization of the latter to nonuniform flows, and it would therefore seem appropriate to refer to it as the "gust" or "hydrodynamic" solution and, more importantly. to use it to represent the incidence turbulence.

We therefore use it to represent the incident turbulence in the present problem of a large flat plate embedded in a turbulent shear flow. Since the gust solution does not satisfy the zero normal velocity boundary condition at the plate, it is necessary to add another solution to cancel this component of velocity. Unlike the gust, this latter solution does not vanish exponentially fast at infinity but rather behaves like a propagating acoustic wave there (Goldstein ${ }^{19}$ ). In other words, the plate is able to "scatter" the nonpropagating motion associated with the gust into a propagating acoustic wave (Ffowcs Williams and $\mathrm{Hall}^{13}$ ).

The problem also possesses an eigensolution associated with the spatially growing instability wave that can propagate downstream from the edge on the inflectional mean-velocity profile (Crighton and Leppington ${ }^{9}$ and Goldstein ${ }^{20}$ ). The solution is therefore not unique! It could be made unique if we required that it remain bounded at infinity (since that would eliminate the eigensolution which grows without bound there). But since the linearization is only valid in the vicinity of the leading edge, it is probably not appropriate to impose a "boundary" condition far downstream in the flow where all sorts of nonlinear effects will have had a chance to intervene (Rienstra ${ }^{36}$ ).
One can therefore look for an alternative way to make the solution unique. This can be done by treating the steady-state solution, which is, of course, the one of interest here, as the long time limit of the solution to an initial-value problem, and then imposing a "causality condition" in the sense that the solution is required to be identically zero before the initial time when the incident disturbance is "turned on" (Crighton and Leppington ${ }^{9}$ ).

But Rienstra36 argued that an initial condition imposed in the distant past may not be relevant to the steady-state solution, since the linearization might only be valid over a relatively short interval of time. One might therefore consider a third way of making the solution unique. This amounts to using the eigensolution to eliminate the leadingedge singularity that appears in both the bounded and causal solutions, f.e. by sattsfying a leadingedge "Kutta condition" (Goldstein 20 ). This may be rationalized by noting that the instability wave represents downstream vortex shedding that could adjust itself to eliminate the singularity in the inviscid solution and thereby prevent any flow separation that would otherwise occur at a very sharp edge.

It is not entirely clear which of these three solutions is correct, but I expect Rienstra's 36 argument is invalid and that imposition of causality is probably appropriate here. Goldstein 19 compared the theory with the data of 01 sen, 35 who measured the sound radiated in one-third-octave frequency bands as a function of the angle from the jet axis in a plane perpendicular to that of the plate. Comparison of experiment and theory is shown in Fig. 2. The top of the figure corresponds to the high-frequency 1 imit where the instability waves are "cut off" and the issues of causality and Kutta condition are irrelevant. However, the lowfrequency causal solution, which is shown at the bottom, is strongly affected by the instability wave. The agreement between experiment and theory is good, but the causal and leading-edge Kutta condition solutions have the same low-frequency limit, and one cannot conclude from this comparison which is correct. However, the bounded solution behaves quite differently in this limit and consequently does not agree with the data.

\section{Sound Generated by Turbulence Interacting With Itself: The Jet-Noise Problem}

Having achleved some success in using linear theory for the turbulence-leading-edge interaction, it is natural to try using it to calculate the sound generated by turbulence interacting with itself, i.e. to deal with the problem of jet noise. I have already pointed out that the ratio rms turbulence velocity to local mean-flow velocity is reasonably small in the region of maximum turbulence level, so that the first requirement for the validity of rapid-distortion theory (see Introduction) is satisfied. However, the interaction time $\tau_{I}$, which in the present context should be taken as the time for the sound generation to occur, is now equal to the decay time $\tau_{\text {decay }}$ of the turbulence, and the second requirement is not. But with no better alternative at hand, we might still attempt to introduce the same small parameter as before, i.e., $U / U$, and carry the corresponding asymptotic expansion to its logical conclusion. Like the more ad 
hoc acoustic-analogy approach, this systematic procedure assures that all appropriate conservation laws will be satisfied and that the acoustic sources will be of the approprlate multipole order. But it seems to have certain advantages over the acoustic analogy in that it provides a "rational" framework for assessing the internal consistency of the various jet-notse analyses. It may also apply to some physically realizable flow, which is hopefully not too different from the real turbulent flow of interest, and finally, it provides a method for identifying acoustic sources and distinguishing acoustic and nonacoustic components of the unsteady motion.

\section{The Basic Equation}

The lowest-order equations are, on the face of it, the same as before, i.e. they are the compressible Rayleigh's equations. It is well known (Betchov and Criminale ${ }^{4}$ ) that the velocity components can be eliminated between these equations to obtain a single equation for the normalized firstorder pressure fluctuation $\Pi_{1} \equiv p_{1} / \rho_{0} C_{0}$. where $p_{1}$ is the actual first-order pressure fluctuation, $p_{0}\left(\vec{x}_{t}\right)$ is the mean-flow density, and $C_{0}\left(\vec{x}_{t}\right)$ is the mean-flow sound speed where the latter two quantities depend only the cross-stream coordinate $\overrightarrow{x_{t}}=$ $\left\{x_{2}, x_{3}\right\}-x_{1}, x_{2}, x_{3}$ denoting Cartesian coordinates with $x_{1}$ in the mean flow direction. This equation can be written symbolically as

$$
\mathrm{L \Pi}_{\mathbf{1}}=0 \text {, }
$$

where $L$ denotes the third-order linear-wave operator

$$
L \equiv \frac{D}{D t}\left(\frac{D^{2}}{D t^{2}}-\nabla \cdot C_{0}^{2} \nabla\right)+2 C_{0}^{2}(\nabla u) \cdot \nabla \frac{\partial}{\partial x_{j}},
$$

$t$ denotes the $t$ ime, and $D / D t=\partial / \partial t+U a / \partial x_{1}$ is the convective derivative based on the mean-flow velocity $U\left(\vec{x}_{t}\right)$.

Since solid boundaries are acoustically irrelevant for the turbulence self-noise problem, it is appropriate to suppose that the flow is defined over all space. Then (for reasons given in the section on solid surface interactions) the "gust" or "hydrodynamic" solution is an appropriate solution of Eq. (1). But this equation also has (spatially growing) instabllity-wave solutions which can exist whenever the mean flow is inflectional (Betchov and Criminale 4 ). Since many investigators have argued (Crighton,6,7 Liu,29 Tam and Chen,38 Haertig, 22 Gaster, Kit, Wygnansk 1,44 and others) that these latter solutions correspond to the experimentally observed large-scale turbulent structures, it would seem appropriate to identify the gust solution with the "fine-grained" (or relatively "finegrained") turbulent motions.

However, there are expertmentally observed, large-scale motions which, on a global basis, seem to bear little resemblance to any motion that can be represented by elther the gust or linear instability-wave solutions. This should come as no surprise, since we have already noted that the 1 inearized solution can at best remain valid over relatively small streamwise distances.

We have seen that the gust solution produces no acoustic radiation at subsonic speeds, and the same can be said for the instability waves (but see below). The asymptotic expansion must therefore be carried to the next order if it is to be used to calculate radiated sound. The second-order normalized pressure fluctuation $\Pi_{2}$ again satisfies a third-order wave equation, but it is more conventent to work with the isentropic density fiuctuation

$$
\Pi \equiv \pi_{2}-\frac{k-1}{2} \pi_{1}^{2} \text {, }
$$

where $k$ is the specific heat ratio. Then $\Pi$ satisfies

$$
L \Pi=Y,
$$

which, except for the inhomogeneous source term

$$
Y \equiv \frac{D}{\partial t} \nabla \cdot \vec{f}-2 \frac{\partial \vec{f}}{\partial x}, \nabla U,
$$

is the same as Eq. (1) for the first-order normalized pressure fluctuation.

Equation (5) is identical to the source term that would be produced by an externally applied fluctuating force per unit mass $\vec{f}=\left\{f_{1}, f_{2}, f_{3}\right\}$ and might therefore be thought of as a dipole-type source, since a fluctuating force produces such a source when there is no mean flow. The force $\vec{f}$ is not, of course, arbitrary but is now given as a quadratic function of the first-order solutions, viz.

$$
f_{1} \equiv \frac{\partial}{\partial x_{j}} u_{1}^{(1)} u_{j}^{(1)}+c_{1}^{2} \frac{\partial}{\partial x_{1}} \pi_{1}, \quad(1, j=1,2,3)
$$

where $u_{1}^{(1)}$ are the first-order velocity fluctuations and $c_{1}^{2}=k \mathscr{R} \mathrm{T}_{1}$ is the first-order square sound speed fluctuation ( $R$ being the gas constant and $T_{1}$ being the first-order temperature fluctuation).

Equations (4) to (6), with some relatively minor differences, were first derived by L111ey, 28 who used the acoustic-analogy approach. The result is now commonly referred to as Lllley's equation. In the present approach, it arises as the equation for the composite second-order pressure fluctuation $\Pi$ with a source term $y$ that involves only firstorder solutions. Since these solutions, which satisfy the homogeneous $\mathrm{Eq}$. (1), have no acoustic fleids at subsonic speeds, while the second-order solution does, our expansion provides a (conceptual if not experimental) mechanism for identifying acoustic and nonacoustic parts of the unsteady motion--but there are some complications.

\section{The Sources of Sound}

The second term in Eq. (6) represents a dipoletypen source due to the temperature fluctuations in the flow (Tester and Morfey ${ }^{42}$ ). While this source is of real significance in actual high-temperature jet exhausts, I will not discuss it in this paper. I concentrate rather on the first term, which, being the divergence of the fluctuating (first-order) Reynolds

stress $u_{i}^{(1)} u_{j}^{(1)}$, corresponds to the source that would be produced by an externally applied fluctuating stress field. It might therefore be 
interpreted, by analogy with the zero-mean-flow case, as a quadrupole-type source.

This latter term can be further decomposed into a number of subsources by separating the first-order solution $u_{i}^{(1)}$ into its gust and inear (spatially growing) instability-wave components and, as before, identifying the gust with the fine-grained turbulent motion. Unfortunately, this procedure cannot be carried to its logical conclusion because the linear instability waves, which grow without bound on a parallel mean flow, will ultimately produce an unbounded source term in Eq. (4). It would then be inapproprlate to use this equation to calculate the acoustic field, since it is its global, and not its local, solutions that must be used in such a calculation. However, the real flow is only locally parallel, and the slowly varying (rather than the parallel-flow) approximation should be used to represent the instablilty waves, as was done by Crighton and Gaster, 8 Tam and Morr 15, 39 and others. Then the source term in Eq. (4) will remain bounded, since the local growth rate of the instablitity wave varies with the thickness of the jet or shear layer (it first increases, reaches a maximum, and then becomes negative as the thickness increases).

However, supersontcally traveling waves can be produced as a by-product of this approximation, and these latter waves will couple to the radiation field (Tam and Morris ${ }^{39}$ ) when the first-order solution is rendered uniformly valid (by using an appropriate singular perturbation procedure, such as the method of multiple scales 34 ). Our previous comment that the first-order solution has no radiation field therefore needs to be qualified. We return to this below, but for now the important point is that the first term in $\vec{f}$ should then describe the sound generation due to the following types of interactions:

(1) Linear instability wave-fine-grained turbulence.

(2) Linear instability wave-linear instability wave.

(3) Fine-grained turbulence-fine-grained turbulence.

This list may be incomplete, of course, or even inappropriate, since as I aiready indicated, there are other types of large-scale motions in the jet that do not seem to be globally representable by either the gust or instability wave.

In any case, it is clear that this list should only be taken as an indication of the types of interactions that can occur and should not be considered to be the result of a rigorous analysis. In fact we shall eventualiy show that nonuniformities in the asymptotic expansion cause these interactions to occur at different asymptotic orders than the present formal expansion would suggest. One might then choose to ignore the list entirely and argue that the experimentally observed turbulent motions should be used in place of the first-order solutions that appear in the source term (5). Which is, in effect, what is done in the acoustic-analogy approach. However. I do not think that it should be dismissed entirely and therefore consider it in some detail.
Its first 1 tem, i.e. the instability wave finegrained turbulence interaction, has only been considered very briefly in the 11 terature ( $F$ fowcs Williams and Kempton, 14 Berman, 3 Liu 29 , and, to myl knowledge, only limtted quantitative results have been obtained for this interaction. It can be thought of as the sound generated by the finegrained turbulence shaking the instability waves and is likely to emerge as an important source mechanism in relatively low Reynolds-number flows.

The instablitty wave-instability-wave interaction may be related to the vortex-pairing events that occur in the inftial mixing region of a highspeed jet. These events can be experimentally enhanced by exciting the jet with an external acoustic source tuned to the most unstable frequency of the shear layer at the nozzle 1ip, as was done by Kibens. 25 He found this to cause a supression of the natural broadband notse of the jet with most of the sound being generated at subharmonics of the excitation frequency. By taking measurements in the near and far fleids, Kibens 25 showed that there was no Doppler shift in frequency, indicating that the sound was generated by nonconvecting sources within the jet, whose locations he subsequently identified with the vortex-pairing locations.

However, quadratic interactions between twodimensional (or between axisymmetric) instability waves produce only subsonically traveling waves on a subsonic parallel mean flow, and these waves do not radiate sound. But the straightforward perturbation analysis of these interactions is (like the stralghtforward nonparallel mean-flow analys is) nonuniformly valid in the streamulse direction (leading to the so-called Kelley45 resonance), and (as in the nonparallel analysts) supersonically traveling waves are produced when the straightforward asymptotic solution is rendered uniformly valid in that direction. The sound field can then be calculated by using a procedure similar to the one used by Huerre and Crighton 23 for the sound generated by the nonlinear saturation of a single instabllity wave. But a more systematic approach might be to adapt the procedure used by Tam and Morris 39 to this case.

\section{The Lighthill Result}

The fine-grained turbulence-fine-grainedturbulence interaction is essentially the mechanism originally considered by Lighthill.26,27 Difficulties such as those discussed in conjunction with instability wave-instablitty-wave interaction may a)so occur when the present perturbation approach is applied to this case. Lighthill's acoustic analogy theory leads to a stationary medium (i.e. classical) wave equation. He suggests that it should be possible to neglect variations in retarded time across the turbulent eddies (or correlation volumes) in this case, since the time $\ell / C_{0}$ $\left(1-M_{c} \cos \theta\right)$ for a sound wave to cross a turbulent eddy will be small compared with the characteristic time $\ell / u^{\prime}$ of the sound source at subsonic jet velocities (Goldstein 77 ). Here $M_{C}$ is the "convection Mach number" of the turbulence, and $\theta$ is the angle between the downstream jet axis and the line connecting the source point and the observation point.

It is a consequence of the linearity of the wave operator on the left side of Eq. (4) that the 
sound radiated by any given turbulent eddy will then be independent of that radiated by any other eddy. Lighthill therefore argued that each eddy should behave like a point quadrupole source moving downstream with the "convection velocity" of the turbulence and that the entire sound field of the jet could then be estimated by calculating the sound radiated by a "typical" turbulent eddy. This picture turns out to be a slight oversimplification and was later corrected by Ffowcs Willlams. 12

In the Lighth11126/Ffowcs williams 12 result, the mean-square pressure $\bar{p}^{2}$ radiated in any proportional frequency band at a fixed source frequency $\Omega$, where $\omega=\Omega\left(1-M_{C} \cos \theta\right)$ is the actual frequency of the sound, behaves like

$$
\overline{p^{2}}-\frac{f(\Omega)}{\left(1-M_{c} \cos \theta\right)^{5}} .
$$

so that its "directivity pattern" is primarily determined by the Doppler factor $\left(1-M_{c} \cos \theta\right)$ raised to the -5 power. These five inverse Doppler factors produce a highly directional radiation pattern at high subsontc Mach numbers--which is remarkably similar to experimental observation.

\section{Solutions of L1lley's Equation}

Solutions of Eq. (4) with $r$ treated as a moving point source can be interpreted as corrections to the Lighthili26/Ffowcs Williams 12 result (7) that account for the effects of the nonuniform surrounding mean flow. A number of us (Mant, 30 Balsa, 1,2 Berman 3 Goldstein, $15,16,21$ Tester and Morfey, 42 Scott, 37 Lilley, 28 etc.) therefore decided to calculate the acoustic radiation from point quadrupole sources moving through transversely sheared mean flows. The relevant solutions usually had to be obtained numerically, but relatively simple closed-form (or nearly closed-form) solutions were obtained in the lowand high-frequency limits $\omega D / U_{J}$ $\ll 1$ and $\omega 0 / U_{J} \gg 1$, respectively, where 0 denotes the jet diameter (see Fig. 1 ) and $U_{J}$ denotes the jet velocity.

Low-frequency solutions were obtained for a round jet with arbitrary mean-velocity proflle by Goldstein 15,16 and Balsa.2 All components of an idealized quadrupole convecting through a stationary medium exhibit directivity patterns given by inverse Doppler factors times sines and costnes of the observation angle. The low-frequency analyses show that only the $x_{1}-x_{1}$ and $x_{1}-r$ quadrupole components (where $r$ is the radial coordinate) retain this property in the presence of a parallel but nonuniform mean flow. The remaining quadrupole components exhlbit directivity patterns given by more-complex formulas involving the complete meanvelocity profile and the location of the sources within the jet (Goldstein ${ }^{16}$ ).

However, the low-frequency analyses uncovered the very surprising result that the mean flow causes certain quadrupole components to emit sound much more efficiently than they otherwise would--the mean-square pressures in the absence and presence of the mean flow being respectively, $0\left(\Omega^{4}\right)$ and $0\left(\Omega^{2}\right)$ as $\Omega \rightarrow 0$. The acoustic field of the $x_{7}-r$ quadrupole, which is the only one of these more efftcient sources that can be expressed in simple ooppler-factor form, is proportional to the local mean-velocity gradient. It is worth noting that this source arises as much from the first member of the source term (5) as from the second, even though the former does not explicitly involve the meanvelocity gradient (Balsa2).

Observed low-frequency jet-nolse directivity patterns therefore depend on complex properties of the jet turbulence and mean flow that are difficult to estimate. But the mathematical results are consistent with the experimentally observed result that they will always be more directional than Lighthill's inverse five Doppler factors would indicate, or, to be more specific, the analytical and experimental results show that the low-frequency sound should be more concentrated on the down-stream axis than Lighthili's result implies, with the onaxis sound being produced by the quadrupoles with one axis in the strearmise direction.

The high-frequency solutions, which were obtained by Lilley, 28 Tester and Burrin, 41 Ba 1 sa, 1,2 Berman, 3 and Goldstein, 17,21 exhibit a "zone of silence" on the downstream jet axis. The acoustic field is exponentialiy small in that region, which is circumferentially asymetric when the jet is nonaxisymmetric and/or the sound source is located off-axis. It will fill the entire range of circumferential angles when $\theta$ is sufficientiy close to the downstream jet axis (see Fig. 3), but will only occupy a limited range of angles (say $\varphi \mathrm{Cmin}<\varphi<$ $\varphi_{\max }$ ) at larger values of $\theta$ (say $\theta_{\mathrm{Cm} i n}$ $<\theta<\theta^{\theta}(\max )$, and finaliy it will disappear completely when $\theta>\theta^{\theta}$ cmax. (See Fig. 4.) These remarks only apply to subsonic isothermal jets with monotonic or neariy monotonic mean-veloctty profiles. A host of complex interference effects may occur when these restrictions are relaxed.

As in geometric optics, the sound propagates along distinct rays in the high-frequency approximation. Only one ray can reach the observer when $\theta>{ }^{\theta} C_{\max }$, but there $w i l l$ be at least two rays reaching the observer when ${ }^{\theta} \mathrm{Cmin}<\theta<\theta^{\theta} \mathrm{Cmax}--$ a direct ray and a ray reflected from the boundary of the zone of silence (Goldstein 21 ). The corresponding sound waves can then interfere, but the interference term will be a rapidly osclllating function of angle and, since all acoustic measurements involve some for of a spatial averaging, may not be experimentally observable.

The mean square pressure radiated in any proportional frequency band of fixed source frequency $\Omega$ will then be the sum of the mean-square pressures for each ray reaching the observer. The result for a convecting quadrupole source, corrected for the Ffowcs Williams 12 effect, is given by (Goldstein ${ }^{21}$ )

$$
\overline{p^{2}} \propto \frac{p_{\infty}^{2} \Omega^{5}\left|\sum_{i, j=1}^{3} a_{i j} v_{j} v_{j}\right|^{2}}{\left(4 \pi R C_{\infty} C_{0}\right)^{2}(1-M \cos \theta)^{2}\left(1-M_{C} \cos \theta\right)^{5}} \Delta(\varphi) \text {, }
$$

where $R$ is the distance between the source point and the observation point shown in Fig. 4, $P_{\infty}$ and $\mathrm{C}_{\infty}$ are the density and sound speeds at infinity, and $M$ is the Mach number based on the mean flow at the source location and the speed of sound at 
infinity. The $Q_{i j}$ denote the relative quadrupole strengths,

$$
\begin{gathered}
v_{1}=q_{0} \cos \lambda, \quad v_{2}=q_{0} \sin \lambda, \quad v_{3}=\cos \theta, \\
q_{0}=\sqrt{\left(\frac{1-M \cos \theta}{c_{0} / C_{\infty}}\right)^{2}}-\cos ^{2} \theta,
\end{gathered}
$$

$\lambda$ is the initial circumferential angle made by the acoustic ray associated with Eq. (8), and $\Delta$ denotes a "circumferential directivity factor" that depends on the circumferential observation angle $\varphi$. the location of the sound source within the jet, and the mean-velocity and temperatures profiles of the jet.

Equation (8) is an exact high-frequency result that applies to jets of any cross section and with any transverse mean-velocity and temperature profiles, but $\lambda$ and $\Delta$ must be calculated by solving a second-order ordinary differential equation in the general case. They are, however, given by relatively simple analytic formulas (Goidstein 21 ) for off-axis sources at arbitrary locations in a circular jet with arbitrary velocity and temperature profiles.

The circumferential directivity factor can be used to study the effect of nonaxisymetric jet velocity and temperature profiles in reducing jet noise below the flight path of a jet aircraft, which is of considerable interest for technological appiication (Von Glahn and Goodykoontz ${ }^{43}$ ). But for the present purpose, it is appropriate to concentrate on the aztmuthal directivity pattern, which is relatively unaffected by this factor.

Equation (8) shows that the inverse-Dopplerfactor exponent is increased from 5 to 7 in the high-frequency limit, since the local mean-flow Mach number and the turbulence-convection Mach number are usually not very different in the regions of peak turbulence intensity. This taken by itself would cause the high-frequency sound (like the lowfrequency sound) to be more directional than Lighthlli's Eq. (7) would predict. If, however, following Balsa, 2 the quadrupole is assumed to be isotropic so that

$$
Q_{1 j}=\delta_{1 j} Q_{0}, \quad(1, j=1,2,3)
$$

Where $\delta_{i j}$ is the Kronecker delta, it follows from Eqs. (9) and (10) that

$$
\left|\sum_{1, j=1}^{3} a_{i j} v_{i} v_{j}\right|^{2}=\left(\frac{1-M_{1} \cos \theta}{c_{0} / c_{\infty}}\right)^{4} 0_{0}^{2} \text {. }
$$

which more than compensates for the additional two Doppler factors in the denominator of $\mathrm{Eq} .(8)$ and produces a net azimuthal directivity pattern that is given by three inverse Doppler factors -- in excellent agreement with the experimentally observed one-third octave directivity patterns.

The interpretation of this result is that the reduced directivity of the high-frequency sound is due to interference between the various quadrupole components. Note that the mean-square pressure is now the product of an azimuthal directivity factor that depends only on $\theta$ and a circumferential directivity factor that depends only on $\varphi$. It is, of course, highly unlikely that the actual quadrupoles will precisely satisfy Eq. (11), but the analys is strongly suggests that quadrupole component interference effects can greatly reduce jet-noise directivity.

\section{Sound Generation Due to Streamwise variations in Mean Flow}

The formal asymptotic expansion in powers of ( $\left.u^{\prime} / U\right)$ can be continued to the third order. At this stage, interactions between the first-order perturbation solution and the streamwise variations in the mean flow will appear in the source term. [Recall that the ratio of the cross-stream to streamwise components of the mean-flow velocity is $O\left(\left(u^{\prime} / U\right)^{2}\right)$. While the first-order solution is $\left.0\left(u^{\prime} / U\right).\right]$ Then, by decomposing the first-order solution into its gust and instability-wave components and making the connection between the gust and fine-grained turbulence that we discussed above, we infer that the source term now describes sound generation due to (a) the fine-grained turbulence interacting with streamwise variations (1.e. spreading) of the mean flow and (b) the instability waves interacting with the streamwise mean-flow variations. The first mechantsm has, to my knowledge, not yet been considered in the iiterature. The second will already be accounted for in the firstorder analysis if the slowiy varying approximation is used to describe the instabifity waves. This mechanism has been analyzed in a more ad hoc fashion by Crow and Champagne, 10 Ffowcs W1111ams and Kempton, 14 Huerre and Crighton, 23 and Liu,29 and in a systematic way by Tam and Morris.39 However. Tam and Morris 39 ultimately conclude that this source is not important at subsonic speeds, which is consistent with the findings of Moore, ${ }^{33}$ who studied the phenomenon experimentally by artificially exciting a jet under conditions that tended to minimize vortex pairing. Unlike Kibens, 25 Moore found that the broadband noise was usually increased rather than suppressed by the external excitation. He concluded that the instability wave, while not radiating noise directly, acted as a conduit through which energy could be transferred to the small-scale turbulent motion.

\section{Concluding Remarks}

Comparisons of the results of the previous section with experiment suggest that the highfrequency solution may remain valid at frequencies that are low enough to include the most energetic portion of the jet-noise spectrum. This might also be true for other more complex turbulent flows. We may therefore be able to calculate the sound generated in such flows by finding the high-frequency solution for a point quadrupole source moving through the appropriate mean flow. Durbinil recently obtained such a solution for a completely general mean flow. Up to now our remarks have been confined to subsontc flows, though much of what has been said also applied to supersonic flows. However, some interesting new phenomena also come into play in these flows. 


\section{REFERENCES}

1. Balsa, T.F., "The far field of high-frequency convected singularities in sheared flows, with application to jet-noise prediction." Journal of Fluid Mechanics. Vol. 74, Part 2, Mar. 23, 1976, pp. 193-208.

2. Balsa, T.F., "The acoustic fleld of sources in shear flow with application to jet noise: convective amplification," Journal of Fluid Mechantcs, Vol. 79, Part 1, Jan. 20, 1977. pp. 33-47.

3. Berman, C.H., "Noise from nonuniform turbulent flows," AIAA Paper 74-2, Jan. 1974.

4. Betchov, R. and Criminale, W.0., Jr., Stability of Parallel Flow. New York:Academic, 1967.

5. Bradshaw, P., Ferriss, D.H., and Johnson, R.F., "Turbulence in the notse-producing region of a circular jet," Journal of Fluid Mechanics. Vol. 19, Part 4, Aug. 1964, pp. 591-624.

6. Crighton, D.G., "Why do the acoustics and the dynamics of a hypothetical mean flow bear on the issue of sound generation by turbulencep" Mechanics of Sound Generation in Flows, ed. E.-A. Muller, Berlin:Springer, 1979, pp. 1-11.

7. Crighton, D.G., "Acoustics as a branch of fluid mechanics," Journal of Fluid Mechanics,

Vol. 106, May 1981, Pp. 261-298.

8. Crighton, D.G. and Gaster, M. "Stability of slowly diverging jet flow," Journal of Fluid Mechantcs, Vol. 77, Part 2, Sept. 24, 1976, pp. 397-413.

9. Crighton, D.G. and Leppington, F.G., "Radiation properties of the semi-infinite vortex sheet: the initial-value problem," Journal of Flutd Mechanics, Vol. 64, Part 2, June 19, 1974, pp. 393-414.

10. Crow, S.C. and Champagne, F.H. "Orderly structure in jet turbulence," journal of Fluid Mechanics, Vol. 48, Part 3, Aug. 16, 1971, pp. 547-592.

11. Durbin, P.A., "High frequency green function for aerodynamic noise in moving media," Journal of Sound and Vibration, Vol. 91, No. 4, Dec. 22, 1983, pp. 519-538.

12. Ffowcs Williams, J.E. "The notse from turbulence convected at high speed," Philosophical Transactions of the Royal Society of London, Series B, Vol. 255, 1963, pp. 469-503.

13. Ffowcs W11liams, J.E. and Hall, L.H., "Aerodynamic sound generation by turbulent flow in the vicinity of a scattering half-plane." Journal of Fluld Mechanics, Vol. 40, Part 4 , Mar. 9, 1970, pp. 657-670.

14. Ffowcs Williams, J.E. and Kempton, A.J., "The noise from the large-scale structure of a jet," Journal of Fluid Mechantcs, Vo1. 84, Part 4 , Feb. 27, 1978, pp. 673-694.
15. Goldstein, M.E., "The low frequency sound from multipole sources in axisymetric shear flows, with applications to jet nolse," Journal of Flutd Mechanics, Vol. 70, Part 3, Aug. 12, 1975, pp. 595-604.

16. Goldstein, M.E.. "The low frequency sound from multipole sources in axisymetric shear flows. Part 2," Journal of Fluld Mechanics, Vol. 75 , Part 1. May 13, 1976, pp. 17-28.

17. Goldstein, M.E., Aeroacoustics. New York: McGraw-Hi11, 1976

18. Goldstein, M.E., "Characteristics of the unsteady motion on transversely sheared mean flows," Journal of Fluid Mechanics, Vol. 84, Part 2, Jan. 30, 1978, pp. 305-329.

19. Goldstein, M.E., "Scattering and distortion of the unsteady motion on transversely sheared mean flows," Journal of Fluid Mechanics, Vol. 91, Part 4, Apr. 27, 1979, pp. 601-632.

20. Goldstein, M.E., "The coupling between flow instabilities and incident disturbances at a leading edge," Journal of Fluid Mechantcs, Vol. 104, Mar. 1981, DD. 217-246.

21. Goldstein, M.E., "High frequency sound emisston from moving point multipole sources embedded in arbitrary transversely sheared mean flows," Journal of Sound and Vibration, Vol. 80, No. 4. Feb. 22, 1982, pp. 499-522.

22. Haertig, J.. "Theoretical and experimental study of wavei ike disturbances in round free jet with emphasis being placed on orderly structure," Mechanics of Sound Generation in Flows, ed. E.-A. Muller, Berilin:Springer, 1979, pp. 167-173.

23. Huerre, P. and Crighton, D.G. "Sound generation by instability waves in a low Mach number flow," AIAA Paper 83-0661, Apr. 1983.

24. Hunt, J.C.R., "A theory of turbulent flow round two-dimensional bluff bodies, "Journal of Fluid Mechanics, Vol. 61, Part 4, Dec. 18, 1973, pp. 625-706.

25. Kibens, V., "Discrete noise spectrum generated by an acoustically excited jet," AIAA Journal, Vol. 18. No. 4, Apr. 1980, pp. 434-441.

26. Lighthill, M.J., "On sound generated aerodynamically: I. General theory," Proceedings of the Royal Society of London Series A, Vol. 211, No. 1107, Mar. 20, 1952, Dp. 564-587.

27. Lighthil1, M.J., "On sound generated aerodynamically: II. Turbulence as a source of sound," Proceedings of the Royal Society of London Sertes A, Vol. 222, No. 1148, Feb. 23, 1954, pp. 1-32.

28. Lilley, G.M., "On the noise from jets," Noise Mechanisms, AGARO CP-131, AGARD: Paris, France, 1974, Pp. 13.1-13.12. 
29. Liu, J.T.C., "Developing large-scale wavelike eddies and near jet noise field," Journal of Fluid Mechanics, Vol. 62, Part 3, Feb. 27, 1974, pp. 437-464.

30. Mani, R., "The Influence of jet flow on jet noise," Journal of Flutd Mechanics, Vol. 73, Part 4, Feb. 24, 1976, pp. 753-793.

31. Moffatt, H.K., "Some developments in the theory of turbulence," Journal of Fluid Mechanics, Vol. 106, May 1981, pp. 27-47.

32. Mohring, W., "Uber Schallwellen in Scherstromungen," Forshritte der Akustik, 1976, pp. 543-546.

33. Moore, C.J., "The role of shear-layer instability waves in jet exhaust noise," Journal of Flutd Mechanics, Vol. 80, Part 2, Apr. 25, 1977, pp. 321-367.

34. Nayfeh, A.H., Perturbation Methods, New York: Wiley, 1973.

35. 01sen, W.A., "Noise generated by impingement of turbulent flow on alrfolls of varied chord, cylinders, and other flow obstructions," AIAA Paper 76-504, July 1976. (NASA TM X-73464).

36. Rienstra, S.W., "Edge influence on the response of shear layers to acoustic forcing." PhD thesis. Technische Hogeschool, Eindhoven. $116 \mathrm{pp}$.

37. Scott, J.N., "Propagation of sound waves through linear shear layer," AIAA Journal, Vol. 17. No. 3, Mar. 1979, pp. 237-244.

38. Tam, C.K.W. and Chen, K.C., "A statistical model of turbulence in two-dimensional mixing layers," Journal of Fluid Mechanics, Vol. 92, Part 2, May 28, 1979, pp. 303-326.
39. Tam, C.K.W. and Morris, P.J., "The radiation of sound by the instability waves of a compressible plane turbulent shear layer," Journal of Fluid Mechanics, Vol. 98, Part 2, May 29, 1980, pp. 349-381.

40. Tennekes, H., Lumley, J.L., 1972. A First Course in Turbulence. Cambridge, Mass: MIT Press.

41. Tester, B.J. and Burrin, R.H., "On sound radiation from sources in parallel sheared jet flows," The Generation and Radiation of Supersonic Jet Exhaust Noise: Studies of Jet Noise Generation and Radiation, Turbulence Structure and Laser Velocimetry, ed. H.E. Plumblee Jr., AFAPL-TR-74-24, 1974, pp. 59-87.

42. Tester, B.J. and Morfey, C.L., "Developments in jet noise modelling--theoretical predictions and comparisons with measured data," Journal of Sound Vibration, Vol. 46, No. 1, May 8, 1976, pp. $79-103$

43. Von Glahn, U. and Goodykoontz, J., "Noise suppression due to annulus shaping as conventional coaxial nozzle," NASA TM-81461, 1980.

44. Gaster, M., Kit, E. and Wygnanski, I., "Large-scale structures in a forced turbulent mixing layer," Journal of Fluid Mechanics, vol. 150, Jan. 1985, pp. 23-39.

45. Kelly, R.E.. "On the stability of an inviscid shear layer which is periodic in space and time, "Journal of Fluid Mechanics, Vol. 27, Part 4, Mar. 20, 1967, pp. 657-689.

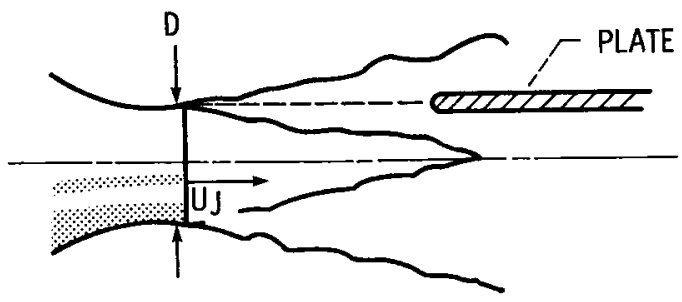

Figure 1. - Plate embedded in turbulent airjet. Note that the leading edge of the plate is positioned in the region of maximum turbulence intensity so that its sound field will exceed the background jet noise by the maximum amount. 


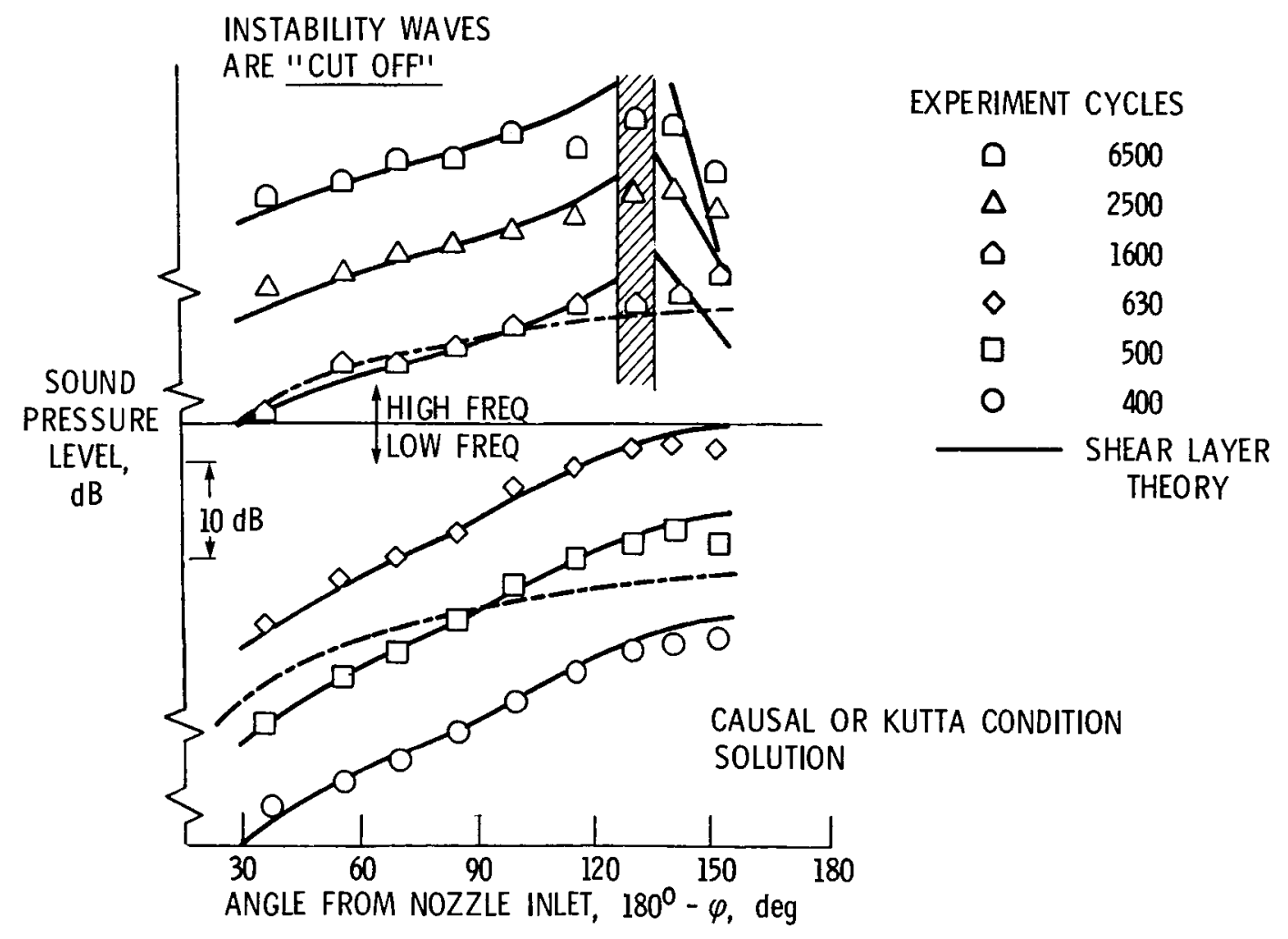

Figure 2. - Comparison of causal or leading-edge Kutta condition solution with data of Olsen (1976) for $U_{j}=7000 \mathrm{ft} \mathrm{s}^{-1}$. 


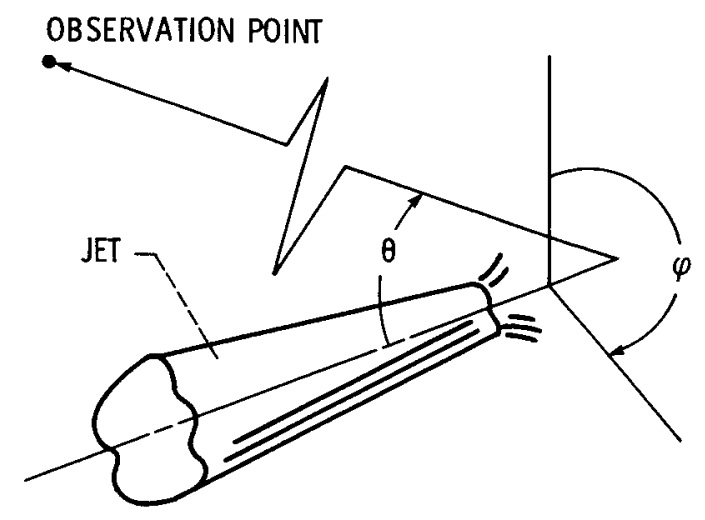

Figure 3. - Coordinates for observation point.

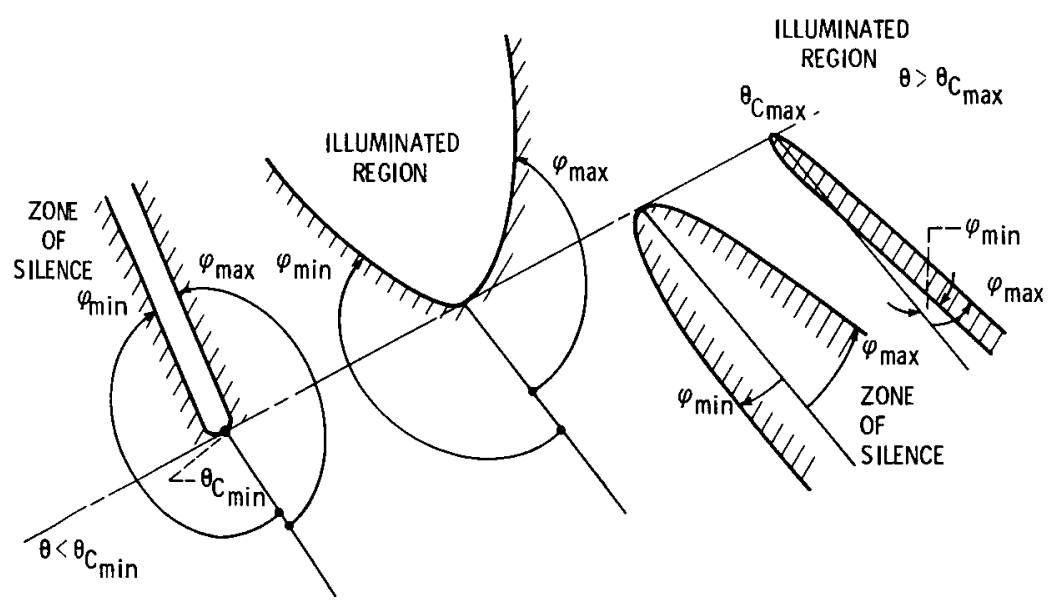

Figure 4. - Asymmetric zone of silence. 


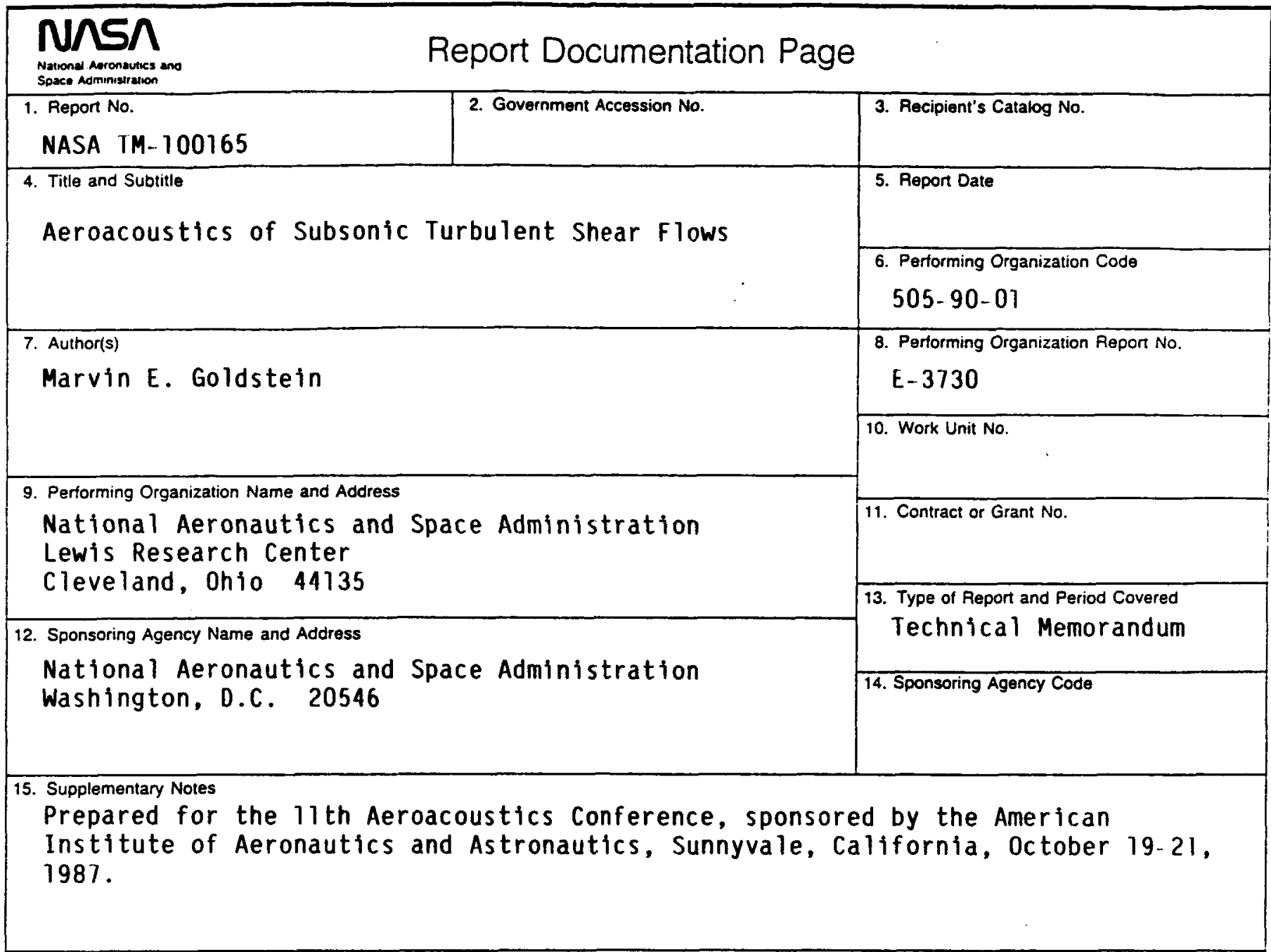

16. Abstract

The paper is a review of sound generation in turbulent shear flows. It empha sizes simultaneous calculation of the turbulent flow along with the resulting sound generation rather than the alternative acoustic analogy approach. The first part of the paper is concerned with solid surface interaction. The second part concentrates on the sound generated by turbulence interacting with itself.

17. Key Words (Suggested by Author(s))

Aeroacoustics

Turbulence

Jet noise
18. Distribution Statement

Unclassified - unlimited

STAR Category 71 